\title{
Eosinophilia in Pleural Effusions: a Speculative Negative Predictor for Malignancy
}

\author{
Fang-Yeh $\mathrm{Chu}^{1,2,3} \&$, Ching-Biau Liou ${ }^{1} \&$, Jen-Tang $\mathrm{Sun}^{4} \&$, Chia-Hao Bei1, Tse- \\ Hsuan Liou' ${ }^{1}$, N-Chi Tan1, Yun-Chieh Yu ${ }^{1}$, Chih-Chun Chang ${ }^{1}$,Tzung-Hai Yen ${ }^{5}$, \\ Ming-Jang $\mathrm{Su}^{1 *}$
}

\begin{abstract}
Background: Eosinophilic pleural effusion (EPE) is an eosinophil count more than $10 \%$ on cytology of pleural samples. Recently, it was reported that malignancy had been the most prevalent cause inducing EPE. Therefore, we conducted an analysis on the prevalence and etiology of EPE and investigated the relationship between EPE and malignancy. Materials and Methods: Data for pleural cell differential count from patients receiving thoracentesis during the period from January 2008 to December 2013 were compared with clinical data and established diagnosis of patients obtained via electronic chart review. Results: A total of 6,801 requests of pleural cytology from 3,942 patients with pleural effusion who had received thoracentesis were available at Far Eastern Memorial Hospital from 2008 to 2013, and of these subjects, 115 (2.9\%) were found to have EPE. The most frequent cause of EPE was malignancy $(33.0 \%, n=38)$, followed by parapneumonic effusions $(27.8 \%$, $\mathrm{n}=32)$, tuberculosis pleuritis $(13.9 \%, \mathrm{n}=16)$, transudate effusions $(12.2 \%, \mathrm{n}=14)$ and the presence of blood or air in pleural space $(10.4 \%, n=12)$. Additionally, an inverse relationship of eosinophilia in pleural fluid was identified in patients with malignancy and EPE. The cut-off eosinophil count in pleural fluid was $15 \%$ for the most accurate discrimination between malignancy and benign disorders in patients with EPE. At the cut-off level, the sensitivity and specificity were $65.8 \%$ and $67.5 \%$, respectively. Conclusions: Pleural fluid eosinophilia was a speculative negative predictor for malignancy, despite the fact that cancers, including lung cancers and metastatic cancers to lung, were the most leading cause of pleural fluid eosinophilia. An inverse correlation was observed between the pleural eosinophil percentage and the likelihood of malignancy in patients with EPE.
\end{abstract}

Keywords: Eosinophilic pleural effusion - lung cancer - malignancy - pleural fluid eosinophilia

Asian Pac J Cancer Prev, 17 (3), 1411-1414

\section{Introduction}

Eosinophilic pleural effusion (EPE) indicated that eosinophil count was more than $10 \%$ in the cytology of pleural fluid (Kalomenidis et al., 2003). To date, the underlying pathogenesis and clinical significance of EPE were yet to be determined. Previous studies had described that a vast number of medical conditions were related to EPE, such as transudate effusions (congestive heart failure, chronic renal failure, hepatic cirrhosis, and so on), parapneumonic effusion, pulmonary embolism, the presence of blood and air in pleural space, parasitic infections, connective tissue diseases and medication reactions (Wysenbeek et al., 1985; Rubins et al., 1996; Kalomenidis et al., 2003; Krenke et al., 2009; Krenke et al., 2011; Kim et al., 2013; Evison et al., 2014; Lin et al., 2014;
Oh et al., 2014; Lin et al., 2015). Besides, it was reported before that certain medical situations such as malignancy and mycobacterium tuberculosis (MTB) infection was less affiliated to the presence of EPE (Adelman et al., 1984). Over the past decades, nevertheless, accumulating evidence had shown that patients with EPE were somewhat associated with malignancy. Some investigations further indicated that there was an inverse correlation between EPE and malignancy (Krenke et al., 2009; Oba et al., 2012). Such discrepancy could be attributed to the varying disease spectrum on the basis of different study populations. The diagnostic role of EPE in malignancy remained to be ascertained. Therefore, we conducted a systemic analysis to survey the prevalence and etiology of EPE as well as the relationship between malignancy and EPE in the Taiwanese population.

${ }^{1}$ Department of Clinical Pathology, Far Eastern Memorial Hospital, New Taipei, ${ }^{2}$ School of Medical Laboratory Science and Biotechnology, Taipei Medical University, ${ }^{3}$ Graduate School of Biotechnology and Bioengineering, Yuan Ze University, Taoyuan, ${ }^{4}$ Department of Emergency, Far Eastern Memorial Hospital, ${ }^{5}$ Division of Nephrology and Clinical Toxicology, Chang Gung Memorial Hospital, Lin-Kou Medical Center, Taoyuan, ${ }^{\star}$ Equal contributors *For correspondence: martinsue@gmail.com 


\section{Materials and Methods}

From January 2008 to December 2013, adult patients (more than 20 years old) who received thoracentesis for cytological examination at Far Eastern Memorial Hospital were enrolled. Baseline characteristics of patients who had EPE were requested, including the age, gender, primary diagnosis of disease and the differential count of pleural fluid, via the electronic medical chart view. EPE was defined as an eosinophilic count of $10 \%$ and more in the pleural fluid specimen. The cytological results of first thoracentesis were adopted for data analysis if a patient had repeated thoracenteses. Besides, the etiological establishment of EPE was based on the patient history, physical examination, image investigation, laboratory and pathological results. The diagnosis of disease was mainly categorized into malignancy, parapneumonic effusion, MTB pleuritis, the presence of blood and air in pleural space and transudate effusions. In patients with EPE related to malignancy, positive findings of malignant cell in histopathology should be available with clinical and image staging after excluding other specific causes of EPE. In patients with EPE related to parapneumonic effusion, typical symptoms and signs such as acute febrile response and pleuritic tenderness, pulmonary infiltrations in image studies and positive microbiological culture in respiratory specimens (including sputum, bronchoalveolar lavage fluid, pleural fluid and pleural biopsy) without other explanation for EPE. For patients diagnosed with MTB pleuritis and EPE, positive culture for MTB in respiratory specimens should be found without alternative causes of EPE. For those found with EPE as well as the presence of blood and air in pleural space, obvious causal relationship of EPE secondary to chest trauma with hemo- and/or pneumothorax or any invasive procedures, including thoracentesis, thoracotomy, thoracoscopy and insertion of pacemaker, should be identified without other explanation. For patients who had certain disorders (such as congestive heart failure, cirrhosis and chronic renal failure) presented with transudate effusions (based on the Light's criteria) and high eosinophilic counts of $10 \%$ and more in the pleural cytology, the results of cytology for malignant cells and microbiology in the pleural fluid should be negative without other explanation of EPE. Furthermore, the subjects with EPE did not meet all the situations mentioned above and thus failed to recognize the etiology of EPE would be assigned as the miscellaneous group.

Statistical analysis was performed using SPSS (version 19.0; SPSS Inc., Chicago, USA) statistical software. All data were shown as mean \pm standard deviation (SD) or median (interquartile range; IQR). Comparison between two clinical variables was either analyzed by student's $\mathrm{t}$ test in normally distributed database, or Mann-Whitney $\mathrm{U}$ test in database without normal distribution. Receiver operating characteristic (ROC) analysis with estimation of the area under ROC curve (AUC) and Youden's index was performed for discrimination of EPEs between malignancy and benign disorders. Statistical significance was considered when a p value was less than 0.05 .

\section{Results}

A total of 6,801 requests of pleural cytology from 3,942 patients with pleural effusion who had received thoracentesis were available at Far Eastern Memorial Hospital from 2008 to 2013, and of these subjects, $115(2.9 \%)$ were found to have EPE. The baseline characteristics of patients with EPE were listed in Table 1. There was no statistical difference of neutrophil and lymphocyte counts in pleural cytology between the groups with malignancy and benign disorders, and lower eosinophil count in pleural cytology was observed in patients with malignancy than those with benign disorders (15\% with IQR of $13-24 \%$ vs. $23 \%$ with IQR of $13-40 \%$, $\mathrm{p}=0.02$ ). The distribution of EPE etiology was shown in Figure 1. The most common cause of EPE was malignancy $(33.0 \%, \mathrm{n}=38)$, followed by parapneumonic effusions $(27.8 \%, \mathrm{n}=32)$, MTB pleuritis $(13.9 \%, \mathrm{n}=16)$, transudate effusions $(12.2 \%, \mathrm{n}=14)$ and the presence of blood or air in pleural space $(10.4 \%, \mathrm{n}=12)$. The miscellaneous group included one case of acute pericarditis, one case of pulmonary embolism and still another one case of unknown origin. Of the subjects who had malignancy with EPE, 26 was lung cancer and 8 had metastatic cancer to lung. The distribution of pleural eosinophil count in patients who had malignancy and benign disorders with EPE was shown in Figure 2. An inverse relationship of eosinophilia in pleural fluid was identified in patients who

Table 1. Baseline Characteristics of Patients who had Malignancy and Benign Disorders with Eosinophilic Pleural Effusion

\begin{tabular}{lcc}
\hline Variables & Malignancy & Benign disorders \\
\hline Age (years) & $65.3 \pm 14.1$ & $66.9 \pm 17.4$ \\
Gender (Male/Female) & $20 / 18$ & $54 / 23$ \\
Pleural cytology (\%) & & \\
Neutrophil & $11(3-24)$ & $12(3-30)$ \\
Lymphocyte & $29(13-53)$ & $24(12-44)$ \\
Eosinophil & $15(13-24)$ & $23(13-40)$ \\
\hline
\end{tabular}

Data were presented as mean \pm standard deviation or median (interquartile range)

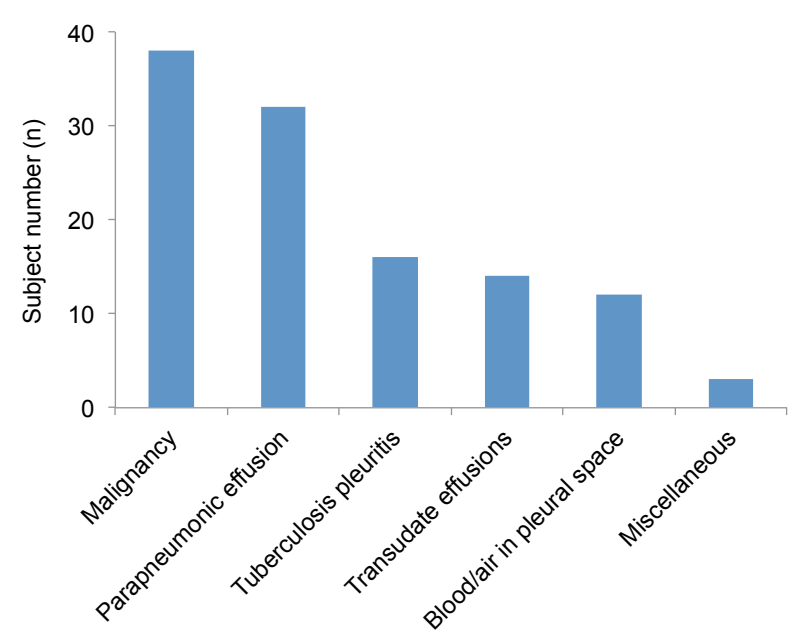

Figure 1. The Etiological Distribution in Patients who had Eosinophilic Pleural Effusion 


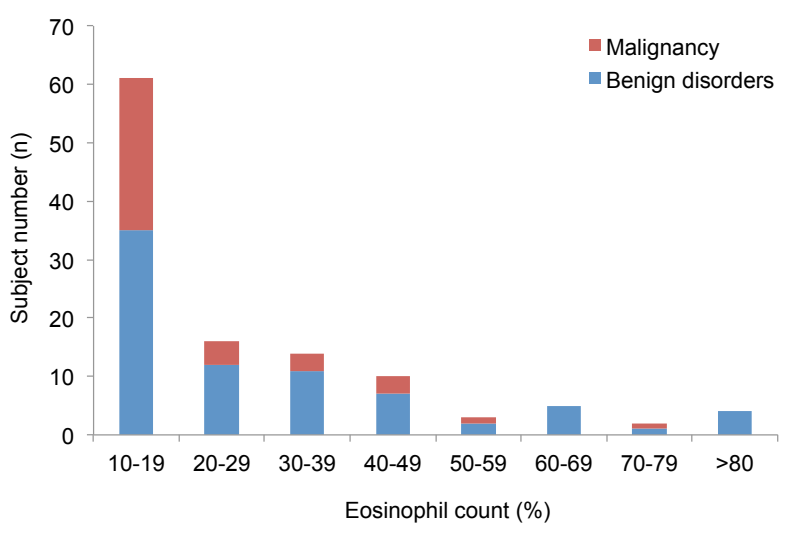

Figure 2. The Distribution of Pleural Eosinophil Count in Patients who had Malignancy and Benign Disorders with Eosinophilic Pleural Effusion

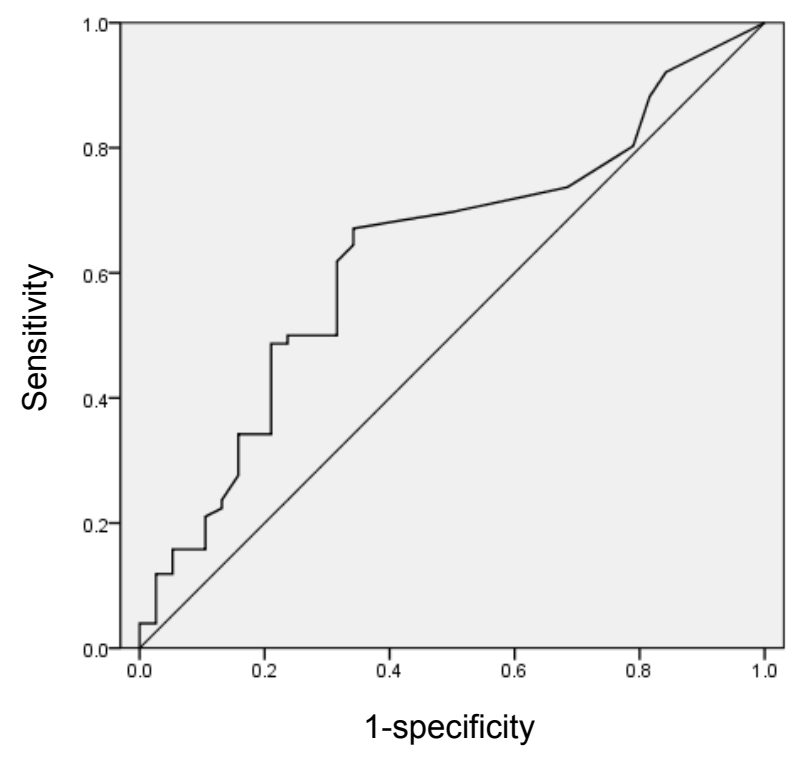

Figure 3. The Receiver Operating Characteristic (ROC) Analysis for Pleural Fluid Eosinophilia as Negative Malignancy Predictor

had malignancy with EPE. The proportion of patients who had malignancy and EPE reduced gradually according to the increasing eosinophilia in the pleural fluid, as $22.6 \%$ in those with an eosinophil count of $10-19 \%, 8.7 \%$ in those with an eosinophil count of $20-49 \%$ and $1.7 \%$ in those with an eosinophil count of 50\% and more, respectively.

The ROC analysis for pleural fluid eosinophilia as negative malignancy predictor was performed and the ROC curve was revealed in Figure 3. The cut-off eosinophil count in pleural fluid was $15 \%$ for the most accurate discrimination between malignancy and benign disorders in patients with EPE. At the cut-off level, the sensitivity, specificity and Youden's index were $65.8 \%$, $67.5 \%$ and 0.333 , respectively. Meanwhile, the AUC was estimated to be 0.631 , suggesting that eosinophil count in pleural effusion was a speculative negative predictor for malignancy in patients with EPE.

\section{Discussion}

Our main finding indicated that malignancy, especially lung cancer and metastatic cancer to lung, was the most leading cause of increased pleural eosinophil count in patients with EPE. Additionally, there was an inverse correlation between the pleural eosinophil count and the probability of malignancy in the population with EPE. Eosinophilia in pleural fluid was an indistinct predictor for freedom from malignancy.

According to literature review, the prevalence of EPE varied from 4.4 to $16.4 \%$ of patients who had fluid accumulation in the pleural space (Oba et al., 2012). Besides, the most accurate cut-off percentage of eosinophil was reported to be 32 to $40 \%$ with Youden index of 0.19 to 0.33 . Contrarily, both the prevalence of EPE (2.7\%) and cut-off level of eosinophil (15\%) were relatively lower in our study. The phenomenon could be explained by different study populations and various timing of pleural fluid collection. The prevalence of idiopathic EPE (0.9\%) was also much lower in our study. One meta-analysis also reported that likelihood of malignancy in EPE was lower than in non-EPE as well as an inverse relationship between the percentage of eosinophil and the proportion of cancer in the group with EPE, implying EPE as a speculative negative predictor for malignancy (Oba et al., 2012). Despite its obscure state in malignancy, however, EPE could not totally exclude possibility of malignancy as EPE subjects with high eosinophil percentage up to more than $80 \%$ were found. Furthermore, the cumulating prevalence of malignant EPE had increased from 7 to $25 \%$ over the recent 4 decades (Oba et al., 2012). The most common cause of EPE had also been reported to be from the presence of blood/air in the pleural space previously shifting to malignancy over these decades. The predictive role of EPE for malignancy remained to be determined.

The wide spectrum of causative factors for EPE formation could be attributed to different pathogenetic mechanisms. To date, at least four mechanisms were proposed for the development of EPE, including: (1) increased eosinophil production in the pleural space; (2) increased eosinophil proliferation in the bone marrow, subsequently increasing eosinophil count in the peripheral blood; (3) increased eosinophilotactic activity and movement from the peripheral blood to the pleural fluid; and (4) increased eosinophil life span in the pleural fluid (Mohamed et al., 2002; Ozkara et al., 2007). Previous studies indicated that several cytokines and growth factors, including interleukin (IL)-3, IL-5 and granulocyte/macrophage colony-stimulating factor (GM-CSF), were responsible for the pathogenesis of eosinophilic recruitment in the pleural cavity (Nakamura et al., 1993; Kalomenidis et al., 2004). Furthermore, it was revealed that certain chemokines, such as vascular cell adhesion molecule (VCAM)-1, chemokine (C-C motif) ligand 5 (CCL5; also regulated on activation, normal $\mathrm{T}$ cell expressed and secreted, RANTES) and CCL26 (also eotaxin-3), were strongly associated with the underlying pathogenesis of EPE post cardiothoracic operations and hemo- and/or pneumothorax (Kalomenidis et al., 2005; Heidecker et al., 2006). In patients who underwent repeated thoracenteses with malignant EPE, a significant increase of tumor necrosis factor (TNF)-alpha and plasminogen-activator inhibitor (PAI)-1 was observed in 
pleural fluid (Chung et al., 2003). In the subgroup of drug smoking-induced EPE, a remarkable increase in pleural vascular endothelial growth factor (VEGF) was found, accompanied with IL-5, IL-6 and IL-8 production (Strong et al., 2003). Currently, it was demonstrated that innate type 2 immunity-dependent and IL-5-mediated immune responses played an essential role in the development of EPE (Kwon et al., 2013).

There were several limitations in our study. First of all, the study design was a retrospective study with limited case number. A large multi-institutional prospective investigation of EPE should be considered. Secondly, the development of EPE due to drug interaction could not be excluded as some of the patients' medication history were incomplete or unavailable and thus make it difficult for detailed survey. Although certain drugs were identified to be associated with EPE formation, the prevalence and mechanism of medication-induced EPE was poorly known. Additionally, the correlation between peripheral blood and pleural fluid eosinophil counts was not investigated due to the lack of information. However, it was reported that no relationship of eosinophil numbers in pleural fluid to that in peripheral blood (Rubins et al., 1996).

In summary, the pleural fluid eosinophilia was an indistinct negative predictor for malignancy. The cancer, including lung cancer and metastatic cancer to lung, was the most leading cause of pleural fluid eosinophilia. Furthermore, an inverse correlation was observed between the pleural eosinophil percentage and the likelihood of malignancy in patients with EPE.

\section{Acknowledgements}

We thankfully acknowledge the financial support from Far Eastern Memorial Hospital (FEMH-2016-C-064).

\section{References}

Adelman M, Albelda SM, Gottlieb J, et al (1984). Diagnostic utility of pleural fluid eosinophilia. Am J Med, 77, 915-20.

Chung CL, Chen YC, Chang SC (2003). Effect of repeated thoracenteses on fluid characteristics, cytokines, and fibrinolytic activity in malignant pleural effusion. Chest, 123, 1188-95.

Evison M, Holme J, Alaloul M, et al (2014). Olanzapine-induced eosinophilic pleuritis. Respir Med Case Rep, 14, 24-6.

Heidecker J, Kaplan A, Sahn SA (2006). Pleural fluid and peripheral eosinophilia from hemothorax: hypothesis of the pathogenesis of EPE in hemothorax and pneumothorax. Am $J$ Med Sci, 332, 148-52.

Kalomenidis I, Light RW (2003). Eosinophilic pleural effusions. Curr Opin Pulm Med, 9, 254-60.

Kalomenidis I, Light RW (2004). Pathogenesis of the eosinophilic pleural effusions. Curr Opin Pulm Med, 10, 289-93.

Kalomenidis I, Stathopoulos GT, Barnette R, et al (2005). Eotaxin-3 and interleukin-5 pleural fluid levels are associated with pleural fluid eosinophilia in post-coronary artery bypass grafting pleural effusions. Chest, 127, 2094-100.

Kim E, Kim C, Yang B, et al (2013). Intrapleural corticosteroid injection in eosinophilic pleural effusion associated with undifferentiated connective tissue disease. Tuberc Respir Dis (Seoul), 75, 161-4.
Krenke R, Nasilowski J, Korczynski P, et al (2009). Incidence and aetiology of eosinophilic pleural effusion. Eur Respir $J, 34,1111-7$.

Krenke R, Light RW (2011). Drug-induced eosinophilic pleural effusion. Eur Respir Rev, 20, 300-1.

Kwon BI, Hong S, Shin K, et al (2013). Innate type 2 immunity is associated with eosinophilic pleural effusion in primary spontaneous pneumothorax. Am J Respir Crit Care Med, 188, 577-85.

Lin SS, Chen YC, Chang YL, et al (2014). Crystal amphetamine smoking-induced acute eosinophilic pneumonia and diffuse alveolar damage: a case report and literature review. Chin J Physiol, 57, 295-8.

Lin Q, Ouyang JS, Li JM, et al (2015). Eosinophilic pleural effusion due to Spirometra mansoni spargana: a case report and review of the literature. Int J Infect Dis, 34, 96-8.

Mohamed KH, Abdelhamid AI, Lee YC, et al (2002). Pleural fluid levels of interleukin-5 and eosinophils are closely correlated. Chest, 122, 576-80.

Nakamura Y, Ozaki T, Kamei T, et al (1993). Factors that stimulate the proliferation and survival of eosinophils in eosinophilic pleural effusion: relationship to granulocyte/ macrophage colony-stimulating factor, interleukin-5, and interleukin-3. Am J Respir Cell Mol Biol, 8, 605-11.

Oba Y, Abu-Salah T (2012). The prevalence and diagnostic significance of eosinophilic pleural effusions: a metaanalysis and systematic review. Respiration, 83, 198-208.

Oh Y, Kim JT, Kim MK, et al (2014). Eosinophilic pleuritis due to sparganum: a case report. Korean J Parasitol, 52, 541-3.

Ozkara SK, Turan G, Basyigit I (2007). Clinicopathologic significance of eosinophilic pleural effusions in a population with a high prevalence of tuberculosis and cancer. Acta Cytol, 51, 773-81.

Rubins JB, Rubins HB (1996). Etiology and prognostic significance of eosinophilic pleural effusions. A prospective study. Chest, 110, 1271-4.

Strong DH, Westcott JY, Biller JA, et al (2003). Eosinophilic "empyema" associated with crack cocaine use. Thorax, 58, 823-4.

Wysenbeek AJ, Lahav M, Aelion JA, et al (1985). Eosinophilic pleural effusion: a review of 36 cases. Respiration, 48, 73-6. 